\title{
The development of automated diffraction tomography
}

\author{
U. Kolb $b^{1,2}$ \\ ${ }^{1}$ Johannes Gutenberg-University Mainz, Duesbergweg 10-14, Mainz, Germany, \\ ${ }^{2}$ Technical University Darmstadt, Schnittspahnstrasse 9, Darmstadt, Germany
}

kolb@uni-mainz.de

Three-dimensional electron diffraction 3DED has turned nowadays into a reliable and promising method used worldwide for the crystal structure analysis of nanoparticles [1]. Since the first attempts to fine scan the electron diffraction space and use the reconstructed reciprocal volume for single crystal structure analysis [2] the method has been adapted in great variety in several labs. In comparison to traditionally applied 3DED methods, where series of oriented diffraction patterns are collected and indexed individually, automated diffraction tomography (ADT) provides major improvements of diffraction data. Scanning the diffraction space while tilting the crystal delivers non-oriented diffraction patterns with reduced dynamical scattering effects, allows to collect the full diffraction information throughout the acquisition range thus providing an enhanced coverage of the expected diffraction intensities as well as improved diffraction intensity determination. The subsequent three-dimensional reconstruction of electron diffraction data provides all information necessary for single crystal structure solution [3]. Dedicated data acquisition strategies and data processing routines allow the investigation of highly beam sensitive material as well as complicated crystallographic features such as the detection and quantitative description of diffuse scattering effects, twinning, superstructures, or modulations.

Crystal structures solved "ab initio" with ADT run through a large number of scientific areas and range from the first structural analysis of barite [3] to complex minerals [4], from the first small organic molecule [5] to large organometallic networks [6], from ZSM-5 single crystals [7] to stacked zeolites and layered silicates [8], from the first pseudo symmetric chalcogenide [9] to complicated oxides covering twinned and modulated materials. Many hitherto unknown crystal structures could be solved by 3DED, several of them new and unexpected structures of often metastable compounds [10].

[1] Gemmi, M., Mugnaioli, E., Gorelik, T.E. Kolb, U., Palatinus, L., Boullay, Ph., Hovmöller, S. Abrahams, J.-P. (2019). ACS Cent. Sci. 5, 1315.

[2] Kolb, U., Gorelik, T., Otten, M. (2008). Ultramic. 108, 763.

[3] Mugnaioli, E., Gorelik, T., Kolb, U. (2009) Ultramic. 109, 758.

[4] Rozhdestvenskaya, I., Mugnaioli, E., Czank, M., Depmeier, W., Kolb, U., Reinholdt, A., Weirich, T. (2010) Mineralogical Magazin,74(1), 159.

[5] Kolb, U., Gorelik, T., Mugnaioli, E., Stewart, A. (2010) Polymer Reviews, 50, 385.

[6] Rhauderwiek, T., Zhao, H., Hirschle, P., Doblinger, M., Bueken, B., Reinsch, H., De Vos, D., Wuttke, S., Kolb, U., Stock, N. (2018) Chem. Sci. 9, 5467.

[7] Mugnaioli, E., Kolb, U. (2014) Microporous and Mesoporous Materials, 189, 107.

[8] Krysiak, Y., Maslyk, M., Silva, B.N., Plana-Ruiz, S., Moura, H.M., Munsignatti, E.O., Vaiss, V.S., Kolb, U., Tremel, W., Palatinus, L., Leitão, A.A., Marler, B, Pastore H. O. (2021), Chem. Mater. 33, 3207.

[9] Birkel, C., Mugnaioli, E., Gorelik, T., Panthöfer, M., Kolb, U., Tremel, W. (2010) J.A.C.S. 132(28) 9881.

[10] Zou, Z., Habraken, W.J.E.M., Matveeva, G., Jensen, A.C.S., Bertinetti, L., Hood, M.A., Sun, Ch-Y. Gilbert, P.U.P.A, Polishchuk, I., Pokroy, B., Mahamid, J., Politi, Y., Weiner, S., Werner, P., Bette, S., Dinnebier, R., Kolb, U. Zolotoyabko, E., Fratzl, P. (2019), Science, 363, 396.

\section{Keywords: electron diffraction, crystal structure analysis, method development}

